\title{
ON THE COEFFICIENTS
}

\section{IN THE QUOTIENT OF TWO ALTERNANTS*}

BY

E. D. ROE, JR.

§1. Introduction.

This paper is complementary to one published in an earlier number of the Transactions. $\dagger$ The notations used there are retained with the following additions :

$$
\begin{gathered}
\| \begin{array}{c}
p_{1} p_{2} \cdots p_{m} \\
\kappa_{1} \kappa_{2} \cdots \kappa_{m}
\end{array}|=|\left[\begin{array}{c}
p_{1} p_{2} \cdots p_{m} \\
q_{1} q_{2} \cdots q_{m}
\end{array}\right]|=|\left[\begin{array}{l}
p \\
q
\end{array}\right] \mid, \\
\left|\begin{array}{c}
\kappa_{1} \kappa_{2} \cdots \kappa_{m} \\
(1)^{j_{1}}\left(1^{2}\right)^{j_{2}} \cdots\left(1^{n}\right)^{j_{n}}
\end{array}\right|=\left[\begin{array}{c}
q \\
\left(1^{i}\right)^{j}
\end{array}\right],
\end{gathered}
$$

where $q_{1}=\kappa_{1}, q_{2}=\kappa_{2}-1, \ldots, q_{m}=\kappa_{m}-m+1$, so that $p$ and $q$ are both partitions of weight $w$;

(2) $\delta_{r} f\left(p_{1}, p_{2}, \cdots, p_{m}\right)=\sum f\left(p_{1}-1, p_{2}-1, \cdots, p_{r}-1, p_{r+1} \cdots p_{m}\right)$,

(3) $\delta_{r} f\left(p_{1}, p_{2}, \cdots, p_{m}\right)=\sum f\left(p_{1}-r_{1}, p_{2}-r_{2}, \cdots, p_{\lambda}-r_{\lambda}, p_{\lambda+1} \cdots p_{m}\right)$,

where

$$
r_{1}+r_{2}+\cdots+r_{\lambda}=r, \quad 1 \leqq \lambda \leqq r, \quad 0 \leqq p_{1}-r_{1} \leqq p_{2}-r_{2} \leqq \cdots,
$$

and where such terms in $\sum$ are excepted as would present the subtraction of the same number from two or more equal $p$ 's ;

(4) $\partial_{r} f\left(p_{1}, p_{2}, \cdots, p_{m}\right)=\partial_{r} f\left(1^{x_{1}} 2^{x_{2}} \ldots r^{x_{r}} \ldots m^{x_{m}}\right)=f\left(1^{x_{1}} 2^{x_{2}} \ldots r^{x_{r}-1} \cdots m^{x_{m}}\right)$;

(5) $\quad \partial_{r} f\left[(1)^{x_{1}}\left(1^{2}\right)^{x_{2}} \cdots\left(1^{m}\right)^{x_{m}}\right]=f\left[(1)^{x_{1}}\left(1^{2}\right)^{x_{2}} \cdots\left(1^{r}\right)^{x_{r}-1} \cdots\left(1^{m}\right)^{x_{m}}\right]$.

* Presented to the Society December 29, 1902. Received for publication May 9, 1904.

†On the Coefficients in the Product of an Alternant and a Symmetric Function vol. 5 (1904), no. 2, pp. 193-213. This paper will be referred to as $I$. 
The subject of investigation is the coefficients $\left|\left[\begin{array}{l}p \\ q\end{array}\right]\right|$ of the symmetric functions $(p)$ in the quotient

$$
\frac{\left|\kappa_{1} \kappa_{2} \cdots \kappa_{m}\right|}{|01 \cdots m|}=\sum\left|\begin{array}{l}
p \\
\kappa
\end{array}\right|(p)=\sum\left[\begin{array}{l}
p \\
q
\end{array}\right] \mid(p) .
$$

A table of such coefficients for weight $w$ is called a quotient table for weight $w$. The following results are obtained: The relations

$$
\left|\left[\begin{array}{l}
p \\
q
\end{array}\right]\right|=\frac{\partial_{r}}{\delta_{r}}\left|\left[\begin{array}{l}
p \\
q
\end{array}\right]\right|, \quad \text { and } \quad \sum_{\kappa}\left|\begin{array}{l:l}
\kappa & \mid \\
p
\end{array}\right| \begin{aligned}
& q \\
& \kappa
\end{aligned} \mid=\begin{aligned}
& 0 \\
& 1
\end{aligned}
$$

according as $\underset{p=q}{p \neq q}$, together with other recurrence formulas, are found in $\S 2$. Generalizations for some of the columns of coefficients are brought out in $\S 3$. In $\S 4$, it is shown that: the table is contained in a triangle and fills up the remainder of the square with the product table ; none of the coefficients is negative; the diagonal line next to the hypotenuse is skew-symmetrical with the corresponding diagonal of the product table; the hypotenuse consists of ones which the table has in common with the product table; the table possesses the invariant property; the coefficients are the conjugates of those of Dr. Taylor's product table; the last line of the table is proved to be symmetrical with respect to its middle element. In addition to the quotients aimed at, the table also gives the determinants $\left\{\kappa_{1} \kappa_{2} \cdots \kappa_{m}\right\}_{a}$, or $\left\{\kappa_{1} \kappa_{2} \cdots \kappa_{m}\right\}_{t}$ in terms of monomial symmetric functions, and by means of a two-fold application it gives the values of the elementary products of weight $w$ in terms of monomial symmetric functions $(\S 5)$.

\section{§ 2. Recurrence Formulas for the $\left|\left[\begin{array}{c}p \\ q\end{array}\right]\right|$.}

1. Dr. Taylor has obtained the following formula,* which has been expressed in the writer's notation, viz.:

$$
\left|\begin{array}{c|c|c}
\kappa & \delta_{r} ! & \kappa \\
\left(1^{i}\right)^{j} & = & \partial_{r} \\
\left(1^{i}\right)^{j}
\end{array}\right|
$$

where the upper operator $\delta_{r}$ applies to the upper complex only, and the lower one $\partial_{r}$ to the lower, and where such terms in the summation are excluded as would make two of the $\kappa$ 's equal, since in this case the corresponding alternant would vanish. By using the partition $q$ of weight $w$, instead of the complex $\kappa$, obtained from it as explained in connection with (1), (7) becomes

$$
\left[\begin{array}{c}
q \\
\left(1^{i}\right)^{j}
\end{array}\right]=\delta_{r}\left[\begin{array}{c}
q \\
\partial_{r} \\
\left(1^{i}\right)^{j}
\end{array}\right]
$$

* On the Product of an Alternant by a Symmetric Function, American Mathematical Monthly, vol. 10 (1903), pp. 119-130. 
where such terms in the sum vanish as cause a smaller number to follow a larger one in the series $q$.

Example: Using $\partial_{3}, \delta_{3}$ as operators, and having the table for weight 4 ,

$$
\left[\begin{array}{c}
12^{3} \\
(1)\left(1^{3}\right)^{2}
\end{array}\right]=\left[\begin{array}{c}
1^{2} 2 \\
(1)\left(1^{3}\right)
\end{array}\right]+\left[\begin{array}{c}
1^{4} \\
(1)\left(1^{3}\right)
\end{array}\right]=1+1=2
$$

2. Expressing (7) in terms of conjugates by the relation (30) of I, and by considering the effect on its conjugate of an operator $\delta_{r}$ on a partition, we find that the operator $\delta_{r}$ goes over into its conjugate $\bar{\delta}_{r}$, whence,

$$
\left|\left[\begin{array}{l}
p \\
q
\end{array}\right]\right|=\frac{\partial_{r}}{\delta_{r}}\left|\left[\begin{array}{l}
p \\
q
\end{array}\right]\right|
$$

Formula (9) reduces a coefficient in the quotient table of weight $w$, to a sum of coefficients in the table of weight $w-r$.

Example: Using $\partial_{2}$ and $\bar{\delta}_{2}$,

$$
\left|\left[\begin{array}{l}
1^{3} 2^{2} \\
2^{2} 3
\end{array}\right]\right|=\left|\left[\begin{array}{c}
1^{3} 2 \\
23
\end{array}\right]\right|+\left|\left[\begin{array}{c}
1^{3} 2 \\
12^{2}
\end{array}\right]\right|=3+2=5,
$$

by the table for weight 5 .

Since the tables read horizontally give the values of the elementary products in terms of the determinants $\left\{\kappa_{1} \kappa_{2} \cdots \kappa_{m}\right\}_{a}=\left[q_{1} q_{2} \cdots q_{m}\right]_{a}$, we obtain from this and (9) the following proposition in determinants:*

$$
a_{r}\left[q_{1} q_{2} \cdots q_{r}\right]=\sum\left[\dot{x_{0}}, q_{1}+x_{1}, q_{2}+x_{2}, \cdots, q_{r}+x_{r}, \cdots, q_{m}\right],
$$

where $x_{0}+x_{1}+x_{2}+\cdots+x_{r}=r, 0 \leqq x_{0} \leqq q_{1}+x_{1}, \leqq q_{2}+x_{2}, \cdots$, and where the same number must not be added to two or more equal indices ( $q$ 's).

3. When the number of elements in $p$ and $q$ is the same, we may find the coefficient by a single reduction by subtracting unity from each element of $p$ and $q$, for the partitions of the reduced coefficient. We have

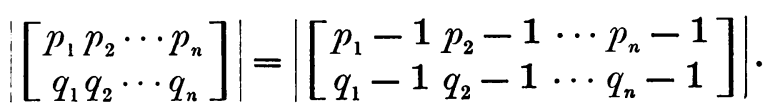

If $n$ be the number of elements in each partition the coefficient of weight $w$ is reduced to one of weight $w-n$. Thus $\left|\left[\begin{array}{c}223 \\ 124\end{array}\right]\right|=\left|\left[\begin{array}{c}122 \\ 13\end{array}\right]\right|=2$, by the table for weight 4 . This depends upon the invariance of the table treated of in $\S 4,8$, , and is there explained.

4. We have also the two following particular recurrence formulas:

$$
\left|\left[\begin{array}{c}
p_{1} p_{2} p_{3} \\
q_{1} q_{2}
\end{array}\right]\right|=\frac{1}{0}+\left|\left[\begin{array}{c}
p_{1}-1 p_{2}-1 p_{3}-1 \\
q_{1}-1 q_{2}-2
\end{array}\right]\right|,
$$

* Cf., MUIr, Vanishing Aggregates of Secondary Minors of a Persymmetric Determinant, Transactions of the Royal Society of Edinburgb, vol. 40 (1902), pp. 511-533. 
where $q_{2} \geqq q_{1}+1$, and where in the first term 0 or 1 is to be taken according as any element of $p$ is or is not greater than $q_{2}$. Similarly

$$
\left|\left[\begin{array}{c}
p_{1} p_{2} p_{3} p_{4} \\
q_{1}^{2}
\end{array}\right]\right|=\frac{1}{0}+\left|\left[\begin{array}{c}
p_{1}-1 p_{2}-1 p_{3}-1 p_{4}-1 \\
\left(q_{1}-2\right)^{2}
\end{array}\right]\right| .
$$

These formulas are derived from two relations given by W. W. Johnson,* taken in connection with the before-mentioned invariance. The relations, using the present notation, are

Examples:

$$
\begin{gathered}
\frac{\left|0 q_{1}+1 q_{2}+2\right|}{012} \mid=t_{q_{2}, q_{1}}+\alpha_{1} \alpha_{2} \alpha_{3} \frac{\left|0 q_{1} q_{2}\right|}{|012|}, \\
\frac{\left|01 q_{1}+2 q_{1}+3\right|}{0123}=t_{q_{1}, q_{1}}+\alpha_{1} \alpha_{2} \alpha_{3} \alpha_{4} \frac{\left|01 q_{1} q_{1}+1\right|}{0123} .
\end{gathered}
$$

$$
\begin{aligned}
& \left|\left[\begin{array}{ll}
2^{2} 3 \\
3 & 4
\end{array}\right]\right|=1+\left|\left[\begin{array}{c}
1^{2} 2 \\
2^{2}
\end{array}\right]\right|=1+1=2, \\
& \left|\left[\begin{array}{ll}
1^{2} 5 \\
3 & 4
\end{array}\right]\right|=0+\left|\left[\begin{array}{c}
4 \\
2^{2}
\end{array}\right]\right|=0+0=0, \\
& \left|\left[\begin{array}{c}
1^{2} 2^{2} \\
3^{2}
\end{array}\right]\right|=1+\left|\left[\begin{array}{c}
1^{2} \\
1^{2}
\end{array}\right]\right|=1+1=2, \\
& \left|\left[\begin{array}{c}
1^{3} 3 \\
3^{2}
\end{array}\right]\right|=1+\left|\left[\begin{array}{c}
2 \\
1^{2}
\end{array}\right]\right|=1+0=1
\end{aligned}
$$

5. From the product table, (I), we have

from the quotient table,

$$
(p) \equiv \sum_{\kappa}\left|\begin{array}{l}
\kappa \\
p
\end{array}\right|\{\boldsymbol{\kappa}\}_{t} ;
$$

$$
\{\kappa\}_{\imath} \equiv \sum_{q}\left\|\begin{array}{l}
q \\
\kappa
\end{array}\right\|(q)
$$

hence

$$
(p) \equiv \sum_{\kappa}\left|\begin{array}{l}
\boldsymbol{\kappa} \\
p
\end{array}\right| \cdot\left(\sum_{q}\left\|\begin{array}{l}
q \\
\kappa
\end{array}\right\|(q)\right) \text {. }
$$

Again from the quotient table

From the product table

$$
a_{p} \equiv \sum_{\kappa}\left\|\begin{array}{l}
p \\
\kappa
\end{array}\right\|\{\kappa\}_{a} .
$$

$$
\{\kappa\}_{a} \equiv \sum_{q}\left|\begin{array}{l}
\kappa \\
q
\end{array}\right| a_{q},
$$

* On a Formula of Reduction for Allernants of the Third Order, American Journal of Mathematics, vol. 7 (1885) pp. 347-352. See also the same author's paper On the Calculation of the Co-Factors of Allernants of the Fourth Order, 1. c. pp. 380-388. 
hence

$$
a_{p} \equiv \sum_{\kappa}\left\|\begin{array}{l}
p \\
\kappa
\end{array}\right\| \cdot\left(\sum_{q}\left|\begin{array}{l}
\kappa \\
q
\end{array}\right| a_{q}\right)
$$

From either of the identities (18) or (21) follows the identity

$$
\sum_{\kappa}\left|\begin{array}{l}
\kappa \\
p
\end{array}\right| \cdot\left\|\begin{array}{l}
q \\
\kappa
\end{array}\right\| \equiv \begin{gathered}
0 \\
1
\end{gathered}
$$

according as $\underset{p=q}{p \neq q}$. From (22) we derive the recurrence formula,

$$
\left|\left[\begin{array}{l}
p \\
q
\end{array}\right]\right|=-\sum_{\kappa}\left|\begin{array}{l}
\kappa \\
q
\end{array}\right| \cdot\left\|\begin{array}{c}
p \\
\kappa
\end{array}\right\|,
$$

where $\kappa$ goes from $123 \cdots m$ to the complex next preceding

Thus

$$
q_{1} q_{2}+1 q_{3}+2 \cdots q_{m}+m-1 \text {. }
$$

$$
\left|\left[\begin{array}{l}
1^{4} 2 \\
1^{3} 3
\end{array}\right]\right|=-(-1-3)=4 \text {. }
$$

By the help of the product table one can by means of this recurrence formula calculate any line of the quotient table beginning at the left of the line. If the quotient table were given the product table might similarly be found by the same relation.

\section{§3. General formulas for the $\left\|{ }_{\kappa}^{p}\right\|$.}

As in the product table, so also in the quotient table it has been possible to generalize certain of the coefficients by columns. Since

$$
\frac{\left|\kappa_{1} \kappa_{2} \cdots \kappa_{m}\right|}{|01 \cdots m-1|}=\left\{\kappa_{1} \kappa_{2} \cdots \kappa_{m}\right\}_{t}=\{\kappa\}_{t}
$$

and since the determinants $\{\kappa\}_{t}$ are given in the product table, we may assume as known the function $\{\kappa\}_{t}$, which gives all the coefficients in the column headed by the complex $\kappa$ or, what is the same thing, by the partition $q$. Let $n_{-r}$ denote the number of different ways of subtracting $r$ units from the complex of exponents $p_{1} p_{2} \cdots p_{m}$ of the symmetric function $(p)$, and let $\left(n_{-r}\right)_{r_{1} r_{1} \cdots r \lambda}$ where $r_{1}+r_{2}+\cdots+r_{\lambda}=r$, denote the number of ways of subtracting $r$ units at $\mathfrak{a}$ time from $p, r_{1}$ from one index, $r_{2}$ from another and so on. Then

Also it is evident that

$$
n_{-r}=\sum\left(n_{-r}\right)_{r_{1} r_{2} \ldots r_{\lambda}} \text {. }
$$

$$
\left\|\begin{array}{l}
p \\
\kappa
\end{array}\right\|=f\left(n_{0}, n_{-1}, n_{-2}, \cdots\right)=f(n),
$$


and the problem of determining the $\left\|{ }_{k}^{p}\right\|$ is a purely combinatorial one. In particular with $p_{1} p_{2} \cdots p_{m}=0^{x_{0}} 1^{x_{1}} 2^{x_{2}} \cdots m^{x_{m}}$,

30)

$$
\begin{gathered}
n_{0}=\left(\begin{array}{c}
x_{1}+\cdots+x_{m} \\
0
\end{array}\right)=1, \quad\left(n_{-2}\right)_{2}=\left(\begin{array}{c}
x_{2}+\cdots+x_{m} \\
1
\end{array}\right), \\
n_{-1}=\left(\begin{array}{c}
x_{1}+\cdots+x_{m} \\
1
\end{array}\right), \quad\left(n_{-2}\right)_{11}=\left(\begin{array}{c}
x_{1}+\cdots+x_{m} \\
2
\end{array}\right), \\
n_{-2}=\left(n_{-2}\right)_{2}+\left(n_{-2}\right)_{11}=\left(\begin{array}{c}
x_{2}+\cdots+x_{m} \\
1
\end{array}\right)+\left(\begin{array}{c}
x_{1}+\cdots+x_{m} \\
2
\end{array}\right), \\
\left(n_{-3}\right)_{3}=\left(\begin{array}{c}
x_{3}+\cdots+x_{m} \\
1
\end{array}\right), \quad\left(n_{-3}\right)_{21}=2\left(\begin{array}{c}
x_{2}+\cdots+x_{m} \\
2
\end{array}\right)+\left(\begin{array}{c}
x_{2}+\cdots+x_{m} \\
1
\end{array}\right)\left(\begin{array}{c}
x_{1} \\
1
\end{array}\right), \\
\left(n_{-3}\right)_{111}=\left(\begin{array}{c}
x_{1}+\cdots+x_{m} \\
3
\end{array}\right), \\
n_{-3}=\left(n_{-3}\right)_{3}+\left(n_{-3}\right)_{21}+\left(n_{-3}\right)_{111}=\left(\begin{array}{c}
x_{3}+\cdots+x_{m} \\
1
\end{array}\right) \\
+2\left(\begin{array}{c}
x_{2}+\cdots+x_{m} \\
2
\end{array}\right)+\left(\begin{array}{c}
x_{2}+\cdots+x_{m} \\
1
\end{array}\right)\left(\begin{array}{c}
x_{1} \\
1
\end{array}\right)+\left(\begin{array}{c}
x_{1}+\cdots+x_{m} \\
3
\end{array}\right),
\end{gathered}
$$

We thus find for the following partitions $q,\{\kappa\}_{t}=F(t)$, and $f(n)$, together with the coefficient which holds for the entire column headed by the partition $q$, as shown by the table on page 007 .

Example: By (35)

$$
\left|\left[\begin{array}{c}
1^{3} 2^{2} \\
1^{2} 5
\end{array}\right]\right|=\left(\begin{array}{l}
4 \\
2
\end{array}\right)=6
$$

\section{§4. The construction and properties of the table.}

1. The partitions are written horizontally and vertically as explained for the product table, I, with complexes of alternants or determinants corresponding to the several partitions heading the columns. The quotient table is read vertically downwards and not horizontally as in the case of the product table. The argument is taken from the horizontal line of complexes at the top of the table in the column headed by the complex $\kappa$. The quotient $\left|\kappa_{1} \kappa_{2} \cdots \kappa_{m}\right| /|01 \cdots m-1|$, is equal to the sum of all the products got by multiplying each coefficient of the column into the symmetric function signified by the partition at the left of the line in which the coefficient occurs.

2. From the nature of division* it is seen that the first term in the quotient

* See MuIR, The Theory of Determinants, \&130, p. 177. 


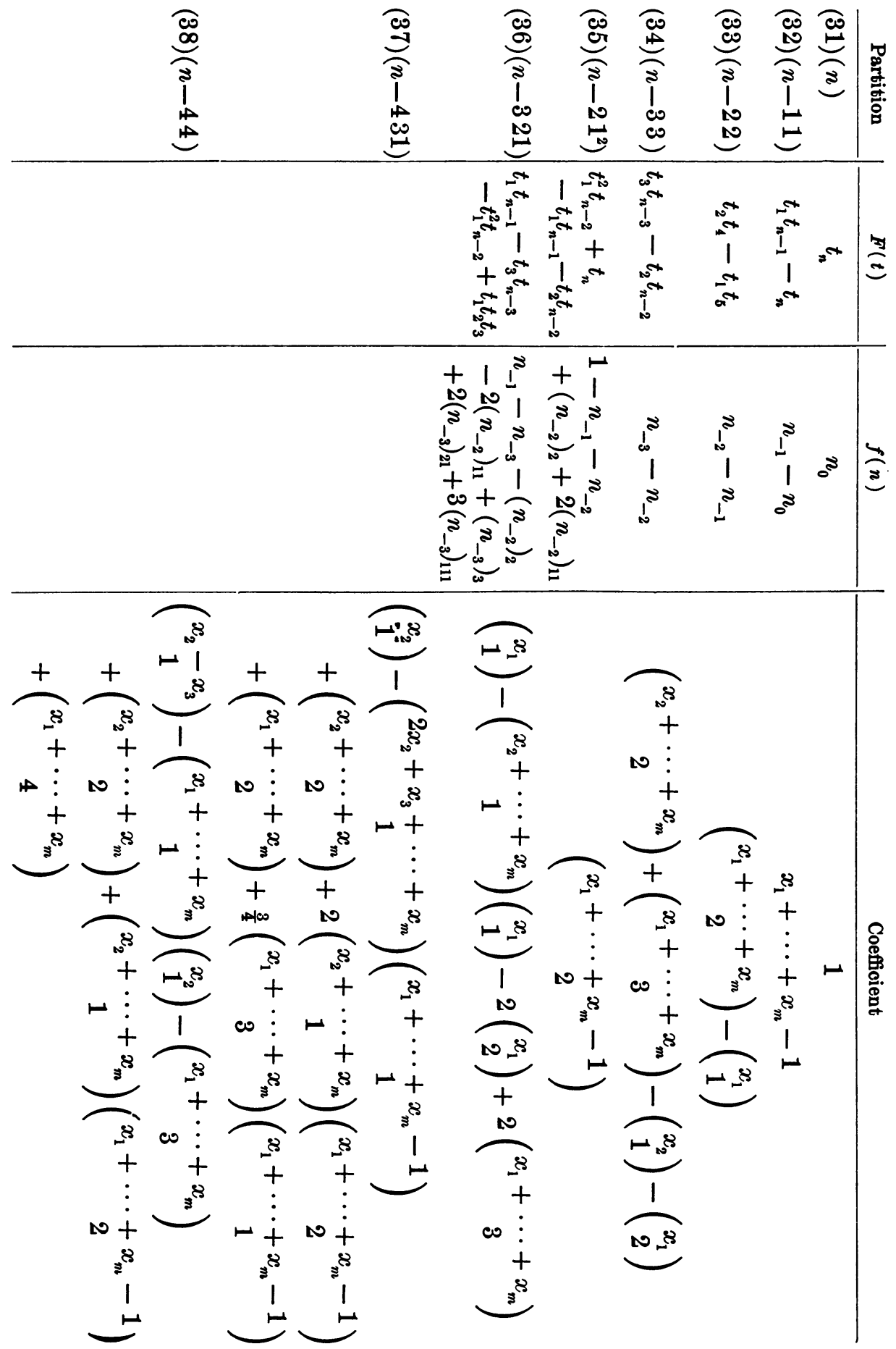


is the symmetric function $(q)$, where $q=\kappa_{1}, \kappa_{2}-1, \cdots, \kappa_{m}-m+1$; whence it follows that

when $p$ precedes $q$, and

$$
\left|\left[\begin{array}{l}
p \\
q
\end{array}\right]\right|=0
$$$$
\left|\left[\begin{array}{c}
p \\
p
\end{array}\right]\right|=1 \text {. }
$$

From (39) and (40) it follows that the table is contained in a triangle which fills up the remainder of the square with the product table; also that the hypotenuse which belongs in common to both tables, consists of ones.

3. Of the coefficients none can be negative. This can be seen from (9) in which no minus sign occurs in the summation. It can also be seen from the standpoint of the coefficients as coefficients in the product of the elementary functions by $|01 \cdots m-1|$. In the formation of this product, units must be laid on the complex in such a way that no inversions of order can arise among the elements of the resulting complexes.

4. The last column consists of ones ; in the next to the last column the coefficient is one less than the number of elements in the partition at the left in the same horizontal line with it. In the third last column the coefficient is equal to the number of combinations of the number of elements taken two at a time, minus the number of elements equal to unity. In the column headed by the partition $\left(n-21^{2}\right)$ the coefficient is equal to the number of combinations of one less than the number of elements in the partition at the left, taken two at a time. These and other like generalizations follow from formulas (31) to (38).

5. The diagonal line next to the hypotenuse is skew symmetrical with the corresponding diagonal of the product table. This follows at once from formula (23). It can also be seen by noticing that the first term of the first remainder in the process of division comes from changing the sign of the alternant next to the hypotenuse. As a consequence of this property no coefficient of the product table in the diagonal next to the hypotenuse can be positive.

6 . It may be recalled that it was shown in I, $\S 4(30)$, that the coefficients are those of Dr. Taylor's product table, when conjugate columns have been interchanged. This also assumes a rearrangement of Dr. Taylor's table according to the writer's method of ordering, as explained in I.

7. The last line of the table is symmetrical with respect to its middle element or elements, as the first line of the product table is symmetrical or skew symmetrical in the same way according as $w$ is odd or even.* In fact

* To prove this we note, by the method of $z 3,2$. I, that all coefficients in the first line will be zero, except those whose column partition consists of $r$ units and the element $m-r$. The value of the coefficient is then, by $\& 3,2$. I,

$$
\left[\begin{array}{c}
m \\
1^{r} m-r
\end{array}\right]=(-1)^{r}
$$




$$
\begin{aligned}
& \left|\left[\begin{array}{c}
1^{m} \\
p_{m} p_{m-1} \cdots p_{2} p_{1}
\end{array}\right]\right|=\left|\left[\begin{array}{c}
1^{m-1} \\
p_{m} p_{m-1} \cdots p_{2} p_{1}-1
\end{array}\right]\right| \\
& \quad+\left|\left[\begin{array}{c}
1^{m-1} \\
p_{m} p_{m-1} \cdots p_{2}-1 p_{1}
\end{array}\right]\right|+\cdots+\left|\left[\begin{array}{c}
1^{m-1} \\
p_{m}-1_{p_{m-1}} \cdots p_{1}
\end{array}\right]\right|, \\
& \left|\left[\begin{array}{c}
1^{m-1} \\
1^{p_{1}-p_{2}} 2^{p_{2}-p_{3}} \cdots m^{p_{m}}
\end{array}\right]\right|=\left|\left[\begin{array}{c}
1^{p_{1}-1-p_{2}} 2^{p_{2}-p_{3}} \cdots m^{p_{m}}
\end{array}\right]\right|
\end{aligned}
$$

$$
\begin{aligned}
+\left|\left[\begin{array}{c}
1^{m-1} \\
1^{p_{1}+1-p_{2}} 2^{p_{2}-1-p_{3}} \ldots m^{p_{m}}
\end{array}\right]\right| & \\
& +\cdots+\left|\left[\begin{array}{c}
1 \\
1^{p_{1}-p_{2}} 2^{p_{2}-p_{3}} \cdots(m-1)^{p_{m-1}+1-p_{m}} m^{p_{m}^{-1}}
\end{array}\right]\right|
\end{aligned}
$$

From (41) and (42), since the lower partitions of the left members and also of the right members term by term are conjugates, it follows, if the property is true for the table of weight $w-1$, it is true for the table of weight $w$. But it is seen to be true when $w=2$, and hence it is true for all values of $w .^{*}$

8. Like the product table it possesses the invariant property. In fact since each is the unique inverse of the other table, the invariance of the quotient table follows from that of the product table. It also appears as follows. We have

$$
\frac{\left|\kappa_{1} \kappa_{2} \cdots \kappa_{m}\right|}{|01 \cdots m-1|}=\{\kappa\}_{t}=[q]_{t} .
$$

If $i_{1} i_{2} \cdots i_{m+r}$ are so taken that

$$
i_{1}, i_{2}-1, i_{3}-2, \ldots, i_{m+r}-m-r-1=0^{r} q_{1} q_{2} \cdots q_{m},
$$

then as an explicit function of $t$,

$$
\frac{\left|i_{1} i_{2} \cdots i_{m+r}\right|}{|01 \cdots m+r-1|}=\{\kappa\}_{t}=[q] .
$$

Hence, in their development in terms of monomial symmetric functions, as far as these functions contain no more than $m$ elements, $\left|i_{1} i_{2} \cdots i_{m+r}\right| /|01 \cdots m+r-1|$ and $\left|\kappa_{1} \kappa_{2} \cdots \kappa_{m}\right| /|01 \cdots m-1|$ must have identical coefficients. In the former the symmetric functions are functions of $m+r$ quantities $\alpha_{1}, \alpha_{2}, \ldots, \alpha_{m+r}$, in the latter they are functions of the $m$ quantities $\alpha_{1}, \alpha_{2}, \ldots, \alpha_{m}$. The latter quotient can be obtained from the former by putting $\alpha_{m+1}=\alpha_{m+2}=\cdots=\alpha_{m+r}=0$

* The value of the conjugate coefficient is

whence

$$
\left[\begin{array}{c}
m \\
1^{m-r-1}(r+1)
\end{array}\right]=(-1)^{m+r+1},
$$

$$
\left[\begin{array}{c}
m \\
1^{r} m-r
\end{array}\right]=(-1)^{m+1}\left[\begin{array}{c}
m \\
1^{m-r-1}(r+1)
\end{array}\right]
$$

which proves the proposition. 
in it. It is also evident that (44) and its development are invariant if all the indices of both numerator and denominator be increased by the same positive integer $\lambda$.

9. If $n_{p}$ denote the number of terms in the symmetric function $(p)$, then $\sum n_{p} \mid{ }_{\kappa}^{p} \|$ is the whole number of terms in the quotient $\left|\kappa_{1} \kappa_{2} \cdots \kappa_{m}\right| /|01 \cdots m-1|$. But this number is equal to the difference product of the $\kappa$ 's divided by the difference product of $0,1,2, \ldots, m-1$ * hence we have the formula

$$
\sum_{p} \frac{n !}{x_{0} ! x_{1} ! x_{2} ! \cdots x_{m} !}\|p\|=\frac{\left(\kappa_{m}-\kappa_{1}\right)\left(\kappa_{m}-\kappa_{2}\right) \cdots\left(\kappa_{2}-\kappa_{1}\right)}{1 ! 2 ! \cdots(m-1) !},
$$

as a farther relation and check for the coefficients of the quotient table.

\section{§5. The Quotient Table as a Table for Elementary Products (as a Product Table).}

A two-fold application of the table gives the elementary products in terms of monomial symmetric functions. Since $\left|\kappa_{1} \kappa_{2} \cdots \kappa_{m}\right| /|01 \cdots m-1|=\{\kappa\}_{t}$, the table gives the values of the determinants $\{\kappa\}_{t}$, in terms of monomial symmetric functions (formula (17)). Also since (I, (41)),

$$
\{\kappa\}_{a}=(-1)^{10} a_{0}^{10}\{\bar{\kappa}\}_{t},
$$

it gives the values of the determinants $\{\kappa\}_{a}$ in terms of monomial symmetric functions.

Thus

$$
\begin{aligned}
{[6]_{a} } & =\left[1^{6}\right]_{t}=\left(1^{6}\right), \\
{[15]_{a} } & =\left[1^{4} 2\right]_{t}=5\left(1^{6}\right)+\left(1^{4} 2\right), \\
{[24]_{a} } & =\left[1^{2} 2^{2}\right]_{t}=9\left(1^{6}\right)+3\left(1^{4} 2\right)+\left(1^{2} 2^{2}\right),
\end{aligned}
$$

with $a_{0}=1$.

Moreover, looked upon as a product table in Dr. Taylor's sense and read horizontally, it gives, by the application of the theorem of corresponding matrices, the elementary products directly in terms of the $\{\kappa\}_{a},(19)$, or, after multiplying the coefficients by $(-1)^{10}$ and using conjugate headings, in terms of the $\{\kappa\}_{t}$. In combination with the product table this gives the elementary products in terms of elementary products and thus leads to the identity (21), or it gives the elementary products developed in terms of the $t$ 's :

$$
a_{p} \equiv \sum_{\kappa}\left|\begin{array}{l|l}
p \\
\kappa
\end{array}\right| \cdot\left(\sum_{q} \mid \begin{array}{l:l}
\bar{\kappa} & t_{q}
\end{array}\right)
$$

* O. H. MitcherL, Note on Determinants of Powers, A merican Journal of Mathematics, vol. 4 (1881), p. 341 . 
By the use of conjugate alternants as headings, it gives directly the product of $|01 \cdots m-1|$ and the elementary symmetric functions.

Examples :

$$
\begin{aligned}
& |012345|\left(1^{4}\right)\left(1^{2}\right)=|123456|+|023457|+|013467| \\
& \begin{aligned}
\left(1^{4}\right)\left(1^{2}\right) & =[24]_{a}+[15]_{a}+[6]_{a} \\
& =\left[1^{2} 2^{2}\right]_{t}+\left[1^{4} 2\right]_{t}+\left[1^{6}\right]_{t} \\
& =\left(1^{2} 2^{2}\right)+4\left(1^{4} 2\right)+15\left(1^{6}\right) \quad[\text { by }(47)] .
\end{aligned}
\end{aligned}
$$

\section{§6. Tables.}

Quotient tables from weight one to weight seven inclusive are given below With each table is also given the product table. The latter consists of the diagonal of ones and the triangle of coefficients above this diagonal, while the quotient table consists of the same diagonal and triangle of coefficients below it. The quotient table is read as explained in $\S 4,1$, the product table as explained in $\mathrm{I}$.

Whight 1.

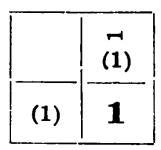

\begin{tabular}{|c|c|c|c|}
\hline & $\underset{\left(1^{3}\right)}{\stackrel{ึ}{n}}$ & $\begin{array}{c}\text { ઠే } \\
\text { (21) }\end{array}$ & $\begin{array}{l}\frac{10}{6} \\
\text { (3) }\end{array}$ \\
\hline (3) & 1 & -1 & 1 \\
\hline (21) & -2 & 1 & 1 \\
\hline$\left(1^{8}\right)$ & 1 & 2 & 1 \\
\hline
\end{tabular}

WhIGHT 2.

\begin{tabular}{|c|c|c|}
\hline & $\begin{array}{c}\dddot{7} \\
\left(1^{2}\right)\end{array}$ & $\begin{array}{c}\Re \\
(2)\end{array}$ \\
\hline$(2)$ & -1 & 1 \\
\hline$\left(1^{2}\right)$ & 1 & 1 \\
\hline
\end{tabular}

\begin{tabular}{|c|c|c|c|c|c|}
\hline & 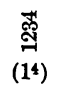 & $\begin{array}{c}\text { 骂 } \\
\left(21^{2}\right)\end{array}$ & $\begin{array}{c}\stackrel{18}{0} \\
(22)\end{array}$ & $\begin{array}{c}0 \\
\stackrel{0}{\sigma} \\
(31)\end{array}$ & 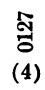 \\
\hline (4) & -1 & 1 & 0 & -1 & 1 \\
\hline (31) & 2 & -1 & -1 & 1 & 1 \\
\hline$\left(2^{2}\right)$ & 1 & -1 & 1 & 1 & 1 \\
\hline$\left(21^{2}\right)$ & -3 & 1 & 1 & 2 & 1 \\
\hline$\left(1^{4}\right)$ & 1 & 3 & 2 & 3 & 1 \\
\hline
\end{tabular}

WEIGHT 3.

\begin{tabular}{|c|c|c|c|c|c|c|c|}
\hline & 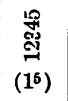 & $\begin{array}{c}\text { 岗 } \\
\left(21^{3}\right)\end{array}$ & $\begin{array}{c}\text { 迎 } \\
\text { 总 } \\
(221)\end{array}$ & $\begin{array}{c}\text { 兽 } \\
\text { (312) } \\
\left(31^{2}\right)\end{array}$ & $\begin{array}{c}\text { 蛋 } \\
(32)\end{array}$ & $\begin{array}{c}\text { 赵 } \\
\text { త్ } \\
\text { (41) }\end{array}$ & 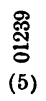 \\
\hline (5) & 1 & -1 & 0 & 1 & 0 & -1 & 1 \\
\hline (41) & -2 & 1 & 1 & -1 & -1 & 1 & 1 \\
\hline (32) & -2 & 2 & -1 & -1 & 1 & 1 & 1 \\
\hline$\left(31^{2}\right)$ & 3 & -1 & -1 & 1 & 1 & 2 & 1 \\
\hline (221) & 3 & -2 & 1 & 1 & 2 & 2 & 1 \\
\hline (218) & -4 & 1 & 2 & 3 & 3 & 3 & 1 \\
\hline (15) & 1 & 4 & 5 & 6 & 5 & 4 & 1 \\
\hline
\end{tabular}

WEIGHT 4.
WEIGHT 5. 
WEIGHT 6.

\begin{tabular}{|c|c|c|c|c|c|c|c|c|c|c|c|}
\hline & 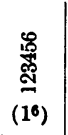 & 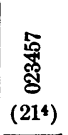 & 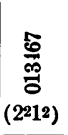 & $\begin{array}{c}\text { 通 } \\
\text { 号 } \\
\left(2^{3}\right)\end{array}$ & $\begin{array}{c}\text { 总 } \\
\text { 鄫 } \\
\left(31^{3}\right)\end{array}$ & 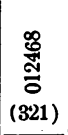 & $\begin{array}{c}\text { 蛋 } \\
\stackrel{3}{8} \\
\left(41^{2}\right)\end{array}$ & $\begin{array}{c}\infty \\
\text { ơ } \\
\text { đ̦ } \\
\left(3^{2}\right)\end{array}$ & 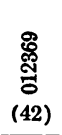 & 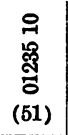 & 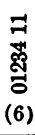 \\
\hline (6) & -1 & 1 & O & 0 & -1 & 0 & 1 & 0 & 0 & -1 & 1 \\
\hline (51) & 2 & -1 & -1 & o & 1 & 1 & -1 & 0 & -1 & 1 & 1 \\
\hline (42) & 2 & -2 & 1 & -1 & 1 & 0 & -1 & -1 & 1 & 1 & 1 \\
\hline (32) & 1 & -1 & 0 & 1 & 1 & -1 & o & 1 & 1 & 1 & 1 \\
\hline (412) & -3 & 1 & 1 & 1 & -1 & -1 & 1 & 0 & 1 & 2 & 1 \\
\hline (321) & -6 & 4 & O & -2 & -2 & 1 & 1 & 1 & 2 & 2 & 1 \\
\hline$\left(31^{3}\right)$ & 4 & -1 & -1 & 0 & 1 & 2 & 3 & 1 & 3 & 3 & 1 \\
\hline$\left(2^{3}\right)$ & -1 & 1 & -1 & 1 & 0 & 2 & 1 & 1 & 3 & 2 & 1 \\
\hline$\left({ }^{(212}\right)$ & 6 & -3 & 1 & 1 & 1 & 4 & 3 & 2 & 4 & 3 & 1 \\
\hline$\left(21^{4}\right)$ & -5 & 1 & 3 & 2 & 4 & 8 & 6 & 3 & 6 & 4 & 1 \\
\hline$\left(1^{6}\right)$ & 1 & 5 & 9 & 5 & 10 & 16 & 10 & 5 & 9 & 5 & 1 \\
\hline
\end{tabular}

WEIGHT 7.

\begin{tabular}{|c|c|c|c|c|c|c|c|c|c|c|c|c|c|c|c|}
\hline & 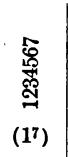 & 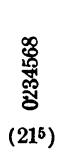 & 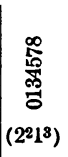 & 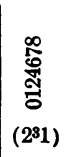 & 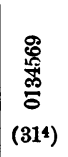 & 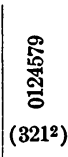 & 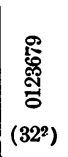 & 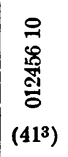 & 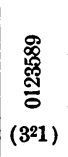 & 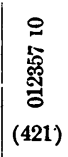 & 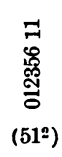 & 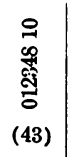 & 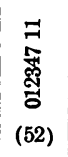 & 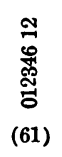 & 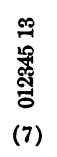 \\
\hline (7) & 1 & -1 & 0 & 0 & 1 & 0 & 0 & -1 & 0 & 0 & 1 & 0 & 0 & -1 & 1 \\
\hline (61) & -2 & 1 & 1 & 0 & -1 & -1 & 0 & 1 & 0 & 1 & -1 & 0 & -1 & 1 & 1 \\
\hline (52) & -2 & 2 & -1 & 1 & -1 & 0 & -1 & 1 & 1 & 0 & -1 & -1 & 1 & 1 & 1 \\
\hline (43) & -2 & 2 & 0 & -1 & -2 & 1 & 1 & 1 & -1 & -1 & 0 & 1 & 1 & 1 & 1 \\
\hline$\left(51^{2}\right)$ & 3 & -1 & -1 & -1 & 1 & 1 & 1 & -1 & 0 & -1 & 1 & o & 1 & 2 & 1 \\
\hline (421) & 6 & -4 & 11 & 0 & 2 & 1 & -1 & -2 & -1 & 1 & 1 & 1 & 2 & 2 & 1 \\
\hline$\left(3^{21} 1\right)$ & 3 & -2 & -1 & 2 & 2 & -1 & -1 & 0 & 1 & 1 & 1 & 2 & $\mathbf{2}$ & 2 & 1 \\
\hline$\left(41^{3}\right)$ & -4 & 1 & 1 & 1 & -1 & -1 & 0 & 1 & 0 & 2 & 3 & 1 & 3 & 3 & 1 \\
\hline$\left(32^{2}\right)$ & 3 & -3 & 2 & -1 & 1 & -1 & 1 & 0 & 1 & 2 & 1 & 2 & 3 & 2 & 1 \\
\hline$\left(321^{2}\right)$ & -12 & 6 & 1 & -2 & -3 & 1 & 1 & 1 & 2 & 4 & 3 & 3 & 4 & 3 & 1 \\
\hline (314) & 5 & -1 & -1 & 0 & $\mathbf{1}$ & 3 & 2 & 4 & 3 & 8 & 6 & 4 & 6 & 4 & 1 \\
\hline (281) & -4 & 3 & -2 & 1 & 0 & 2 & 3 & 1 & 3 & 6 & 3 & 4 & 5 & 3 & 1 \\
\hline$\left(2^{2} 1^{8}\right)$ & 10 & -4 & 1 & 2 & 1 & 6 & 5 & 4 & B & 11 & 6 & 6 & 7 & 4 & 1 \\
\hline$\left(21^{6}\right)$ & -6 & 1 & 4 & 5 & 5 & 15 & 10 & 10 & 11 & 20 & 10 & 9 & 10 & 5 & 1 \\
\hline$\left(1^{7}\right)$ & $\mathbf{1}$ & 6 & 14 & 14 & 15 & 35 & 21 & 20 & 21 & 3i) & 15 & 14 & 14 & 6 & 1 \\
\hline
\end{tabular}

